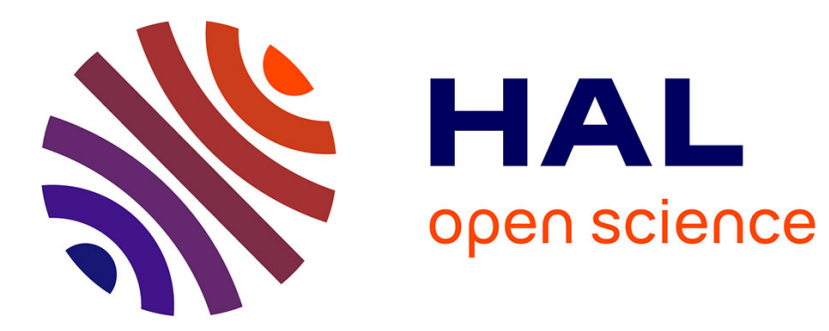

\title{
High harmonic attosecond pulse train amplification in a free electron laser
}

B W J Mc Neil, N R Thompson, D J Dunning, B Sheehy

\section{To cite this version:}

B W J Mc Neil, N R Thompson, D J Dunning, B Sheehy. High harmonic attosecond pulse train amplification in a free electron laser. Journal of Physics B: Atomic, Molecular and Optical Physics, 2011, 44 (6), pp.65404. 10.1088/0953-4075/44/6/065404 . hal-00604053

\section{HAL Id: hal-00604053 https://hal.science/hal-00604053}

Submitted on 28 Jun 2011

HAL is a multi-disciplinary open access archive for the deposit and dissemination of scientific research documents, whether they are published or not. The documents may come from teaching and research institutions in France or abroad, or from public or private research centers.
L'archive ouverte pluridisciplinaire HAL, est destinée au dépôt et à la diffusion de documents scientifiques de niveau recherche, publiés ou non, émanant des établissements d'enseignement et de recherche français ou étrangers, des laboratoires publics ou privés. 


\title{
High harmonic attosecond pulse train amplification in a Free Electron Laser
}

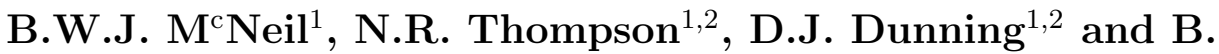 \\ Sheehy ${ }^{3}$ \\ 1 SUPA, Department of Physics, University of Strathclyde, Glasgow, G4 0NG, UK. \\ 2 ASTeC \& Cockcroft Institute, STFC Daresbury Laboratory, Warrington, WA4 \\ $4 \mathrm{AD}, \mathrm{UK}$. \\ 3 Collider-Accelerator Department, Brookhaven National Laboratory, Upton, New \\ York 11973, USA. \\ E-mail: b.w.j.mcneil@strath.ac.uk
}

\begin{abstract}
It is shown using three dimensional simulations that the temporal structure of an attosecond pulse train, such as that generated via High Harmonic generation in noble gases, may be retained in a Free Electron Laser amplifier through to saturation using a Mode Locked Optical Klystron configuration. At wavelengths of $\sim 12 \mathrm{~nm}$, a train of attosecond pulses of widths $\sim 300$ attoseconds with peak powers in excess of $1 \mathrm{GW}$ are predicted.
\end{abstract}

PACS numbers: 41.60.Cr, 42.65.Ky, 42.65.Re

Submitted to: J. Phys. B: At. Mol. Opt. Phys. 


\section{Introduction}

Attosecond pulses from High Harmonic generation in noble gases can been used to great effect in capturing and controling ultra-fast events in atomic and molecular physics [1, 2]. While many techniques require single attosecond pulses, methods that utilise trains of attosecond pulses, as generated in the normal mode of operation of an $\mathrm{HH}$ source, have also been employed to both capture and control electronic processes within atoms and molecules $[3,4,5,6,7,8]$

High-gain free-electron lasers (FELs) operating at similar wavelengths typically have longer pulse durations but offer peak powers several orders of magnitude greater than the $\mathrm{HH}$ sources. If a FEL is seeded by and amplifies a $\mathrm{HH}$ source, the initial $\mathrm{HH}$ power $P_{0}$, is exponentially amplified as a function of distance through the FEL interaction region as $P(z) \approx P_{0} \exp \left(z / \sqrt{3} l_{g}\right)$ where $l_{g}=\lambda_{u} / 4 \pi \rho$ is the nominal gainlength, $\rho$ is the FEL scaling parameter that determines the FEL coupling strength and efficiency and $\lambda_{u}$ is the period of the undulator [9]. However, due to the relative propagation of the radiation pulses through the amplifying electron bunch, such amplification of the $\mathrm{HH}$ source tends to 'wash out' the $\mathrm{HH}$ attosecond pulse train structure [10].

Several techniques have been proposed to achieve attosecond pulse durations in FELs (see e.g. $[11,12,13,14,15])$. The widths of the light pulses generated are limited by the coherence length of the FEL interaction $l_{c}=\lambda_{r} / 4 \pi \rho$, where $\lambda_{r}$ is the resonant FEL wavelength [9], and pulse widths tend to have durations $l_{c} / c \gtrsim 2 \mathrm{fs}$ at the $\sim 12 \mathrm{~nm}$ wavelengths considered here.

An alternative method, that applies the concepts from mode-locked cavity lasers in FEL amplifiers, has also been proposed [16] that may generate a train of pulses with durations not restricted by the FEL coherence length $l_{c}$. In this technique, a series of spatiotemporal shifts are introduced between the radiation and the co-propagating electron bunch that define a set of axial radiation modes. The spatiotemporal shifts are achieved by delaying the electron bunch using magnetic chicanes inserted between undulator modules. Introducing a modulation of the electron beam properties at the mode spacing periodically suppresses or enhances the FEL interaction at different longitudinal positions along the beam, so that a train of equally spaced pulses develops aligned with the regions of strongest FEL interaction. In the frequency domain this is equivalent to the modes developing sidebands that overlap neighbouring modes, allowing mode-locking to occur and is analogous with mode-locking in conventional cavity lasers [17].

The spectrum generated by the XUV mode-coupled SASE system of [16] has a modal structure with mode-spacing $\Delta \omega$ similar to the spacing of harmonics in a $\mathrm{HH}$ source. Equivalently, the temporal structure of the two systems form an attosecond pulse train. By matching the spectral/temporal structures of a mode-coupled FEL amplifier to an $\mathrm{HH}$ seed, it may therefore be possible to amplify the $\mathrm{HH}$ seed to saturation while retaining its attosecond train structure. Furthermore, it should be possible to 


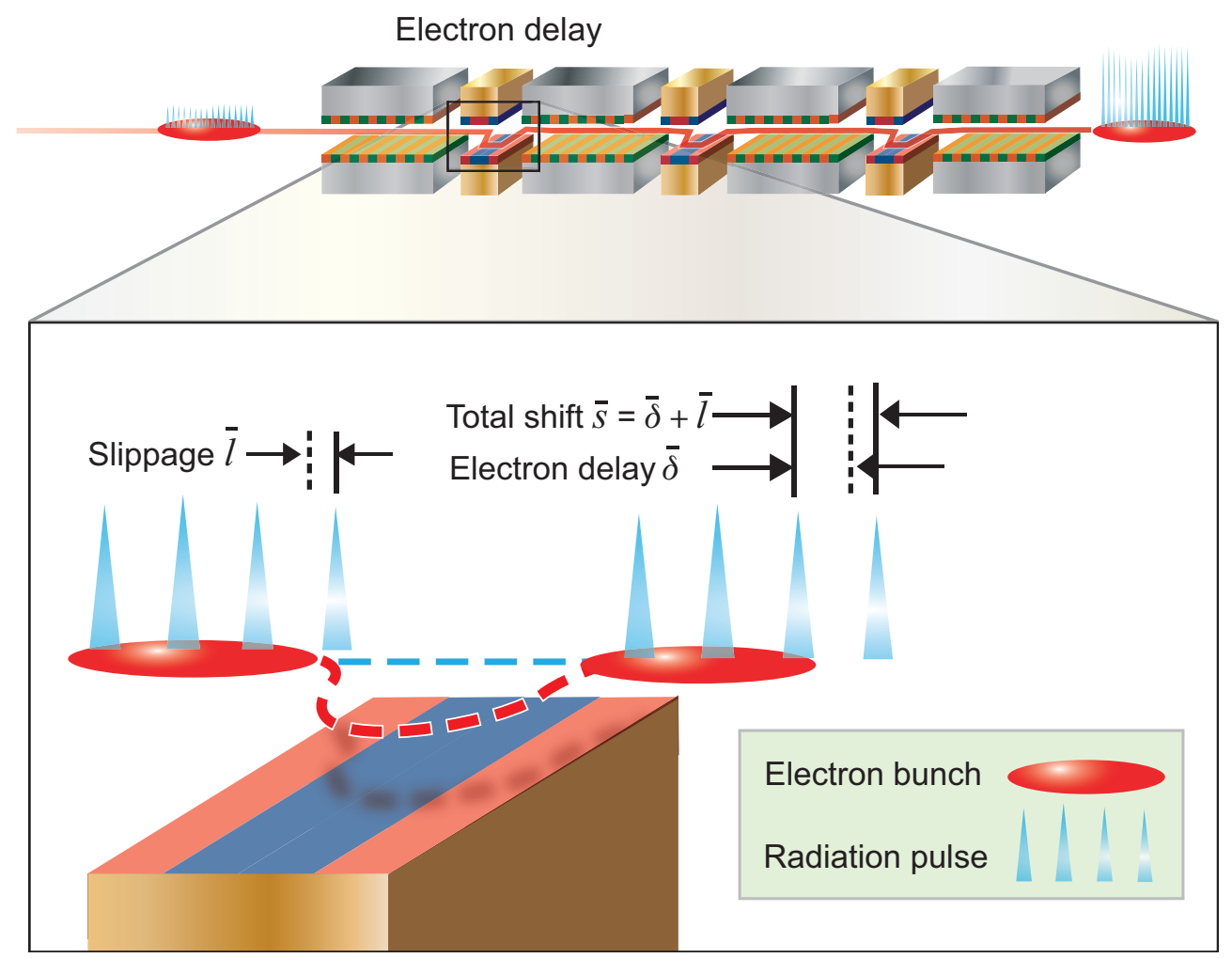

Figure 1. Schematic of the mode-coupled amplifier FEL interaction operating with an $\mathrm{HH}$ seed. The inset shows detail of the electron delay.

achieve this without the need for introducing a modulation at the mode spacing as the $\mathrm{HH}$ attosecond seed already comprises a pulse train structure. In this paper it is demonstrated via $1 \mathrm{D}$ and $3 \mathrm{D}$ numerical simulations that this is the case.

\section{Mode generation in an amplifier}

The spatio-temporal shifts that generate the equally spaced modal structure in the amplifier spectrum are achieved by periodically delaying the electron bunch using magnetic chicanes between undulator modules [16], as shown schematically in fig. 1.

The notation used here follows that of [16]. The total propagation distance of a radiation wavefront ahead of the electron bunch per module in units of the cooperation length $l_{c}$ is $\bar{s}=s / l_{c}=\bar{l}+\bar{\delta}$, where $\bar{l}$ is the similarly scaled slippage occurring in the undulator and $\bar{\delta}$ is that within the chicane. The scaled FEL radiation frequency is defined as $\bar{\omega}=\left(\omega-\omega_{r}\right) / 2 \rho \omega_{r}$ where $\omega_{r}$ is the resonant FEL frequency. The slippage enhancement factor is defined as $S_{e}=\bar{s} / \bar{l}$. In [16], a solution is obtained for the one dimensional wave equation describing the field evolution of a small, constant electron source term for a series of $N$ undulator-chicane modules. For $S_{e}>1$ the spectrum takes the form of a sinc-function envelope (the single undulator module spectrum) modulated 
by a frequency comb centered at the scaled resonant frequency $\bar{\omega}$, with mode separation $\Delta \bar{\omega}=2 \pi / \bar{s}$ corresponding to $\Delta \omega=2 \pi / T_{s}$, where $T_{s}=s / c=\bar{s} l_{c} / c$ is the time taken for radiation to travel the slippage length. The number of modes under the central peak of the spectrum is shown to be $N_{0}=2 S_{e}-1$.

The delay provided by a four dipole chicane is of the order of its $R_{56}$. The HH pulses are separated by one half the drive laser wavelength i.e. $\sim 400 \mathrm{~nm}$, so the $R_{56}$ of the slippage enhancing chicanes are of this order. By comparison, a bunch compression chicane used prior to electron injection into the FEL has an $R_{56}$ typically of several $\mathrm{cm}$, and the slippage enhancing chicanes placed between the undulator modules are seen to be weaker by several orders of magnitude. Any CSR disruption caused by these chicanes can therefore assumed to be negligibly small.

\section{Amplification of an $\mathrm{HH}$ seed}

The temporal profile of the $\mathrm{HH}$ seed is a comb of attosecond pulses separated by half the wavelength of the drive laser $\lambda_{d}$ and modulated by the drive laser power envelope. The spectral and temporal structure of the radiation generated by the undulator-chicane system is matched to that of the HH seed by setting $\bar{s}=\bar{\lambda}_{d} / 2$, where $\bar{\lambda}_{d}=\lambda_{d} / l_{c}$ is the scaled drive laser wavelength.

For a typical FEL parameter of $\rho=2 \times 10^{-3}$, similar to that for an FEL operating in the XUV, then for undulator modules of 8 periods, $\bar{l}=0.201$. The value of the scaled slippage generated by the chicanes is chosen to be $\bar{\delta}=0.616$ so that $\bar{s}=\bar{\lambda}_{d} / 2$, matching the undulator-chicane system to the spectral and temporal structure of the $\mathrm{HH}$ seed. This gives a slippage enhancement factor of $S_{e}=4.0625$.

The HH seed was modelled in a similar way to that described in [10] with a FEL resonant wavelength chosen to be the 65 th harmonic of the drive laser wavelength of $\lambda_{d}=805 \mathrm{~nm}$ (e.g. Ti:Sapphire)i.e. $\lambda_{r}=\lambda_{d} / 65$. The HH field was constructed by first summing over a weighted harmonic series modulated by an envelope determined by the Gaussian drive laser centered at $t=t_{0}$ and of width $\sigma_{d} \approx 3 \mathrm{fs}$ :

$$
E_{H H} \propto \exp \left(-\frac{\left(t-t_{0}\right)^{2}}{2 \sigma_{d}^{2}}\right) \sum_{h} \mathcal{E}_{h} e^{-i\left(\omega_{h}\left(t-t_{0}\right)+\phi_{h}\right)}
$$

where $h=1,3,5, \ldots, \omega_{h}=h \omega_{d}$ are the odd harmonics of the drive laser frequency and $\phi_{h}$ and $\mathcal{E}_{h}$ represent the phases and amplitudes of individual harmonic components.

In a typical $\mathrm{HH}$ spectrum, the harmonic yield drops quickly over the first few harmonic orders, then much more slowly over a 'plateau region', up to a wavelength and intensity dependent cutoff order, beyond which the yield drops precipitously. Both the amplitude of particular harmonics and their relative phases are sensitive functions of the experimental conditions. We consider here a somewhat idealized HH source while retaining its essential features. The field amplitude dependence is assumed to be of the 
form:

$$
\mathcal{E}_{h}=\left(\frac{\eta_{p}^{h_{m} / h_{p}}}{\eta_{m}}\right)^{\frac{h_{p}}{h_{m}-h_{p}}} \exp \left(-\frac{\ln \left(\eta_{p} / \eta_{m}\right)}{h_{m}-h_{p}} h\right)
$$

where $h_{m}=95$ is the maximum harmonic, $h_{p}=11$ is the harmonic where the plateau region begins, $\eta_{p}=5 \times 10^{-6}$ is the harmonic yield efficiency of the HH process at the beginning of the plateau and $\eta_{m}=5 \times 10^{-7}$ that at the maximum harmonic. We choose this form to approximate the slow decline in harmonic yield of one to two orders of magnitude between the beginning of the plateau and the cutoff that has been observed in experiments [18]. A different smoothly falling function or small variations of individual harmonic amplitudes from this dependence does not significantly alter the attosecond character of the seed.

Most numerical FEL simulation codes, like those used in this paper, average both the electron motion and the radiation field locally within the pulses over a radiation wavelength. The Nyquist theorem then limits the bandwidth of frequencies that can be properly modelled to $\omega_{r} / 2<\omega_{r}<3 \omega_{r} / 2$ [10] which, for the resonant harmonic $h=65$ considered here, corresponds to an harmonic range $33<h<95$, all of which fall within the harmonic plateau region. We include only these harmonic orders in the simulation.

The attosecond character of the seed is sensitive to variations in the relative phases, $\phi_{h}$, of the harmonics [19]; when these phases vary, the pulse train can have more structure than that of the 'clean' pulse train of fig. 2, in which it was assumed $\phi_{h}=0$ for all harmonic orders. While we use the phase-locked seed of fig. 2 in our simulations below for clarity, we have also varied these phases and run the simulations using a more structured seed and find, unsurprisingly, that the amplified output also has more structure, although a well defined pulse train is retained.

The system is modelled using a 1D code similar to that described in [20]. The $\mathrm{HH}$ seed is injected into the amplifying undulator-chicane modules with a cold electron beam. Note that no energy modulation of the electron beam was used. This is different from the mode-locked SASE case of [16] which starts up from noise and requires a beam energy modulation at the mode spacing $\Delta \bar{\omega}$ to generate an isolated train of equally spaced pulses. Here, the HH seed is modelled as a set of in-phase modes, hence it consists of a train of equally spaced pulses in the temporal domain. It will be seen that while this temporal structure is retained, there occurs a broadening of the individual pulses. The pulse durations attained are in agreement with the scaling observed in [16] for the pulse width, $\tau_{p} \propto 1 / \sqrt{N_{0}}$, where $N_{0}$ is the number of amplified modes.

After 22 undulator-chicane modules the amplified scaled radiation power and spectrum is as shown in Fig. 3. It is seen that the HH seed is amplified and the comb of temporal spikes is retained, but with increased pulse widths as discussed. This number of modules corresponds to a scaled FEL interaction length of $22 \times \bar{l} \approx 4.4$. This is just prior to saturation where scaled powers of $|A|^{2} \approx 1$ are achieved. However, the temporal structure of the pulses begins to break up at saturation. (Note that the dispersive strength of the chicanes $D=R_{56} / 2 l_{c} \approx 10 \bar{\delta} / 6$ reduces the gain length as 

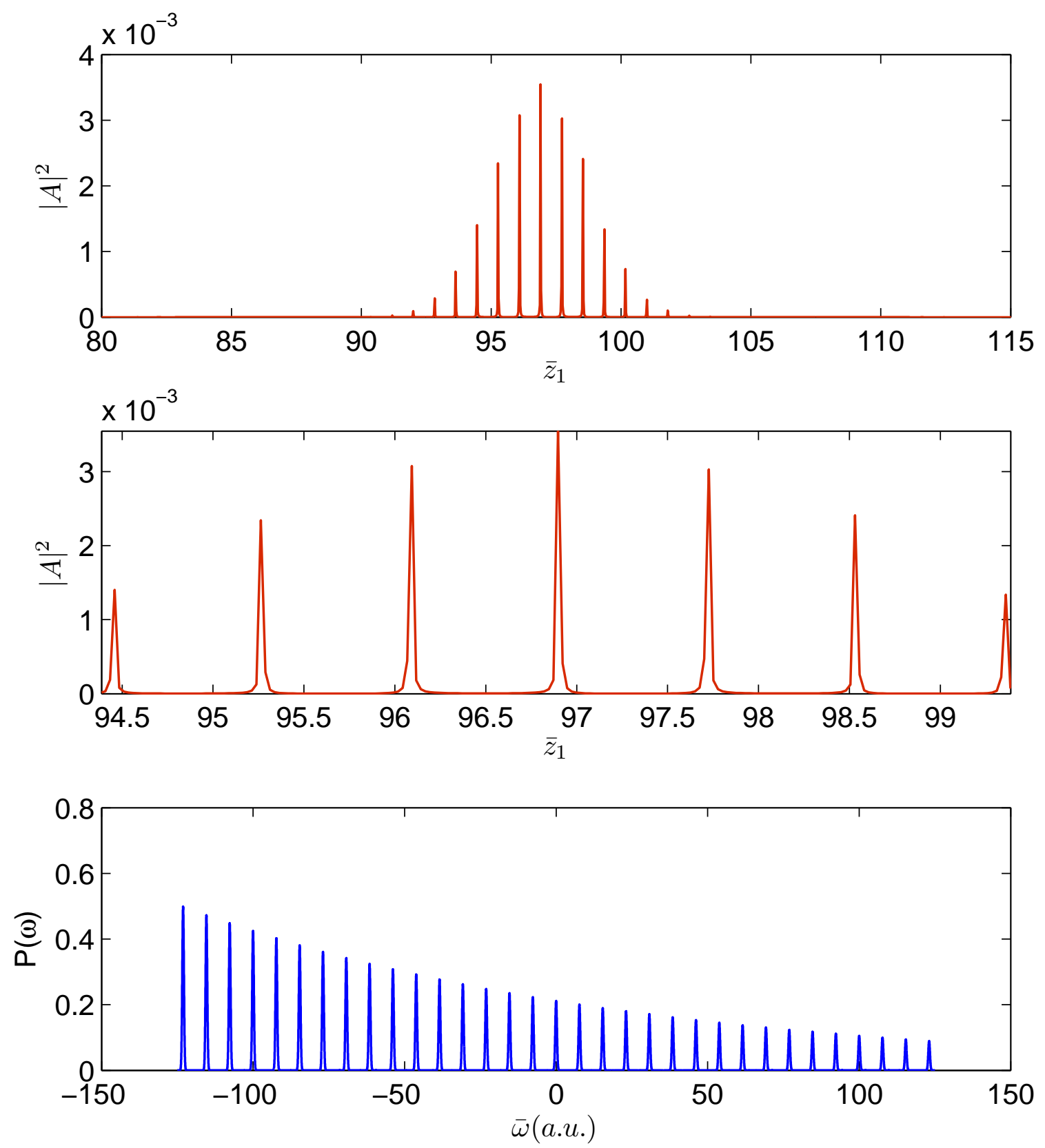

Figure 2. Scaled longitudinal intensity profile top and middle (detail) as a function of the scaled distance within the pulse $\bar{z}_{1}$ measured in cooperation lengths, and scaled spectral power distribution for the $\mathrm{HH}$ seed (bottom).

discussed in [16]).

\section{Optimising the system}

In [16] it is demonstrated that the pulse widths in mode-locked SASE simulations are proportional to $1 / \sqrt{N_{0}}$, this is in agreement with an analogous relation from modelocked conventional cavity lasers.

The maximum bandwidth of the amplified radiation spectrum is that of the single 

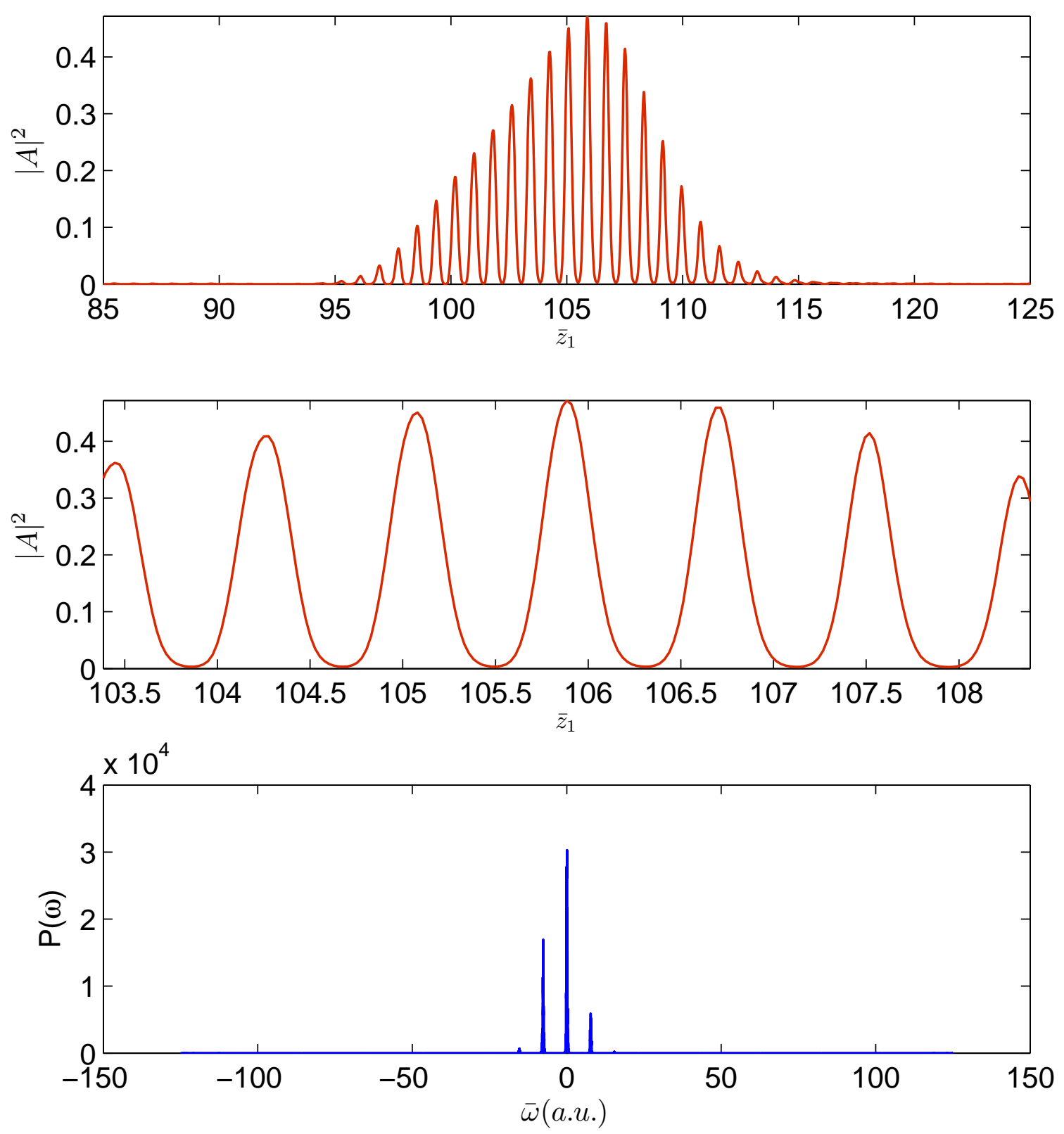

Figure 3. Scaled longitudinal intensity profile (top and middle) and scaled spectral power distribution of the amplified HH radiation for $\bar{l}=0.201\left(S_{e}=4.0625\right)$, after 22 undulator-chicane modules.

undulator module spectrum, with the minimum number of optical cycles per pulse approximately equal to the number of undulator periods per module. In order to attain narrower amplified $\mathrm{HH}$ pulses, the bandwidth of the system (and hence number of modes amplified) is increased by reducing the number of undulator periods per module. To reduce $\bar{l}$ while continuing to satisfy the condition $\bar{s}=\bar{\lambda}_{d} / 2$, the value of $\bar{\delta}$ is increased to keep $\bar{s}$ constant. The system is simulated for a case with the number of undulator periods per module set to four. The corresponding value of the slippage enhancement 
factor is $S_{e}=8.125$.

The spectral power distributions for the two cases $S_{e}=4.0625$ and $S_{e}=8.125$ are shown in Figs. 3 and 4 respectively. It is seen that the width of the spectral envelope of the amplified radiation is increased with reduced undulator module length $\bar{l}$. The number of modes, $N_{0}$, under the central peak of the spectrum increases, narrowing the temporal duration of the pulses. The variation of the peak intensity and pulse width with scaled distance through the undulator are plotted in Fig. 5 for the two cases $S_{e}=4.0625(\bar{l}=0.201)$ and $S_{e}=8.125(\bar{l}=0.101)$. For both cases, there is an initial rapid broadening since only the modal content that falls under the envelope of the single undulator spectrum is amplified. The pulse durations continue to increase more slowly during further amplification. This slow pulse broadening effect is attributed to slippage in the undulators. For $S_{e}=8.125,34$ undulator-chicane modules are required to produce the same amplification of the 22 module $S_{e}=4.0625$ case. This corresponds to a reduction in the total undulator length from 4.4 to 3.4 , which can be attributed to more effective seeding since a broader spectral range of the seed is amplified, and reduced gain length due to an increased number of chicanes.

For larger values of $S_{e}$, the broadening of the modal sinc-function envelope becomes restricted by the Nyquist limit.

\section{Energy Spread}

The effects of energy spread on the interaction have been investigated using the 1D model by introducing a relative gaussian energy spread of width $\sigma_{\gamma} / \gamma=\rho / 10=0.02 \%$ for the case $S_{e}=8.125$. The results close to saturation are plotted in Fig. 6, which is the equivalent of Fig. 4. While the cold beam case took 34 undulator-chicane modules, the case here took 42 modules. Note that the temporal and spectral structure of the radiation power remains largely unaffected by the energy spread. Larger energy spreads have also been simulated with the main effect being that a larger number of modules are required to achieve saturation.

\section{Results of 3D Simulations}

The system has also been modelled using the three-dimensional FEL simulation code Genesis 1.3 [21]. The main results of the 1D simulations are reproduced using the 3D code and show amplification of an $\mathrm{HH}$ seed pulse retaining its pulse train structure. The Genesis simulation code models both the radiation field and the electron beam in 3D and requires careful matching of the electron beam to the undulator/chicane lattice structure which additionally contains an electron beam quadrupole focussing lattice to maintain good electron-radiation field coupling through the long structure. The focussing lattice was optimised for normal FEL interaction in the absence of the chicane fields and it was found that this set-up also worked well when the chicanes were included to generate the radiation modes matched to the $\mathrm{HH}$ seed. 

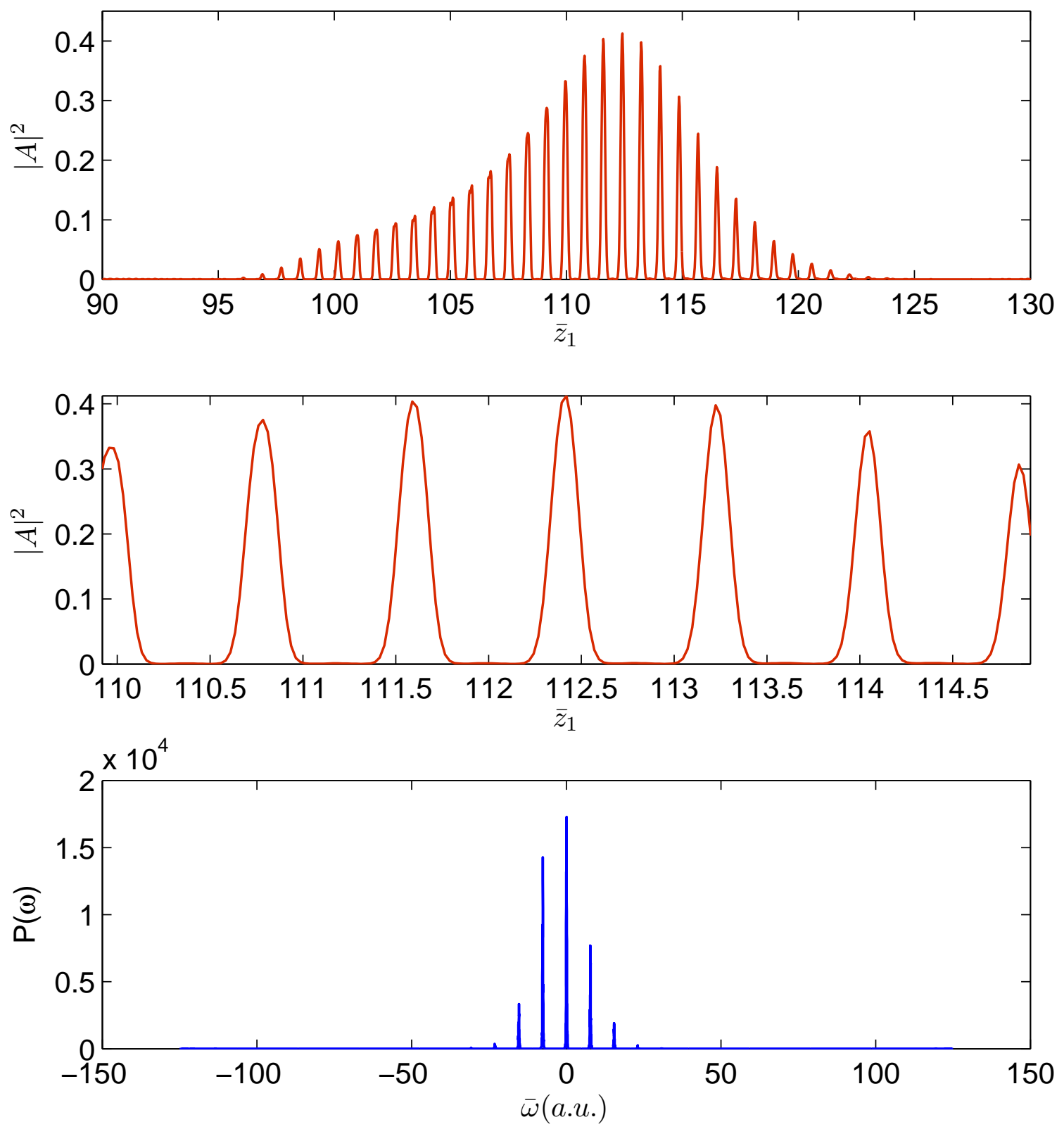

Figure 4. Scaled longitudinal intensity profile (top and middle) and scaled spectral power distribution of the amplified HH radiation for $\bar{l}=0.101\left(S_{e}=8.125\right)$, after 34 undulator-chicane modules.

Similar parameters as for the XUV mode-coupled FEL amplifier of [16] were used (i.e. without electron beam modulation) including an energy spread of width $\sigma_{\gamma} / \gamma=0.01 \%$. The seed power and spectrum show the attosecond pulse train structure and frequency comb, as seen in Fig. 7. The peak power of the seed is $3 \mathrm{MW}$ in an envelope of $\sigma_{d} \approx 25 \mathrm{fs}$. (This peak power is a reasonable estimate of what can be expected from $\mathrm{HH}$ sources in the present to near future [22] and can be reduced by an order of magnitude while still retaining a good amplified pulse train 

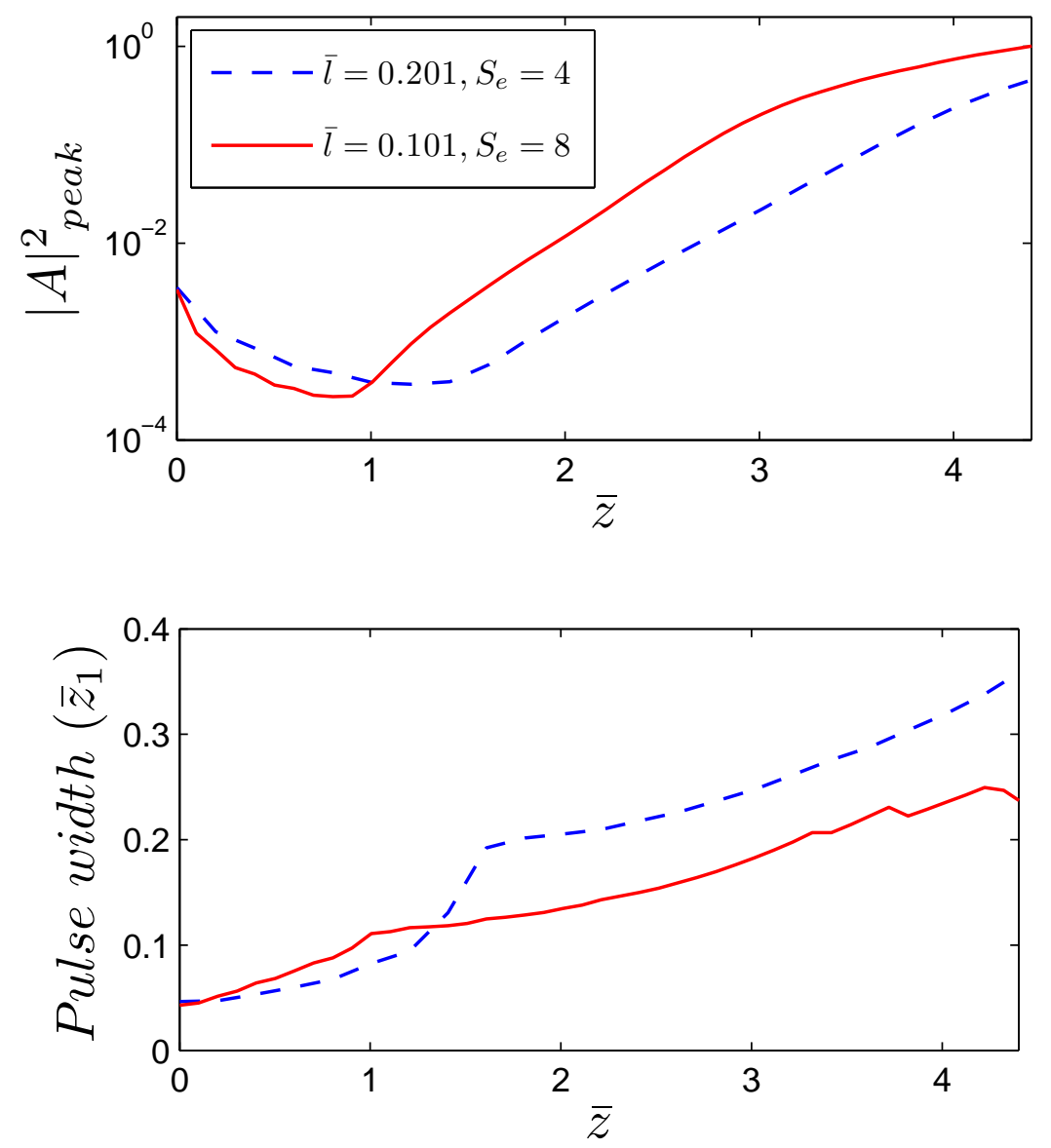

Figure 5. Scaled peak intensity (top) and pulse width in units of $l_{c}$ (bottom) plotted against the scaled interaction length, $\bar{z}[16]$, for the two cases $S_{e}=4.0625(\bar{l}=0.201)$ and $S_{e}=8.125(\bar{l}=0.101)$. It is seen that by reducing the number of undulator periods per module, both the pulse duration and the gain length are reduced.

structure that dominates competing SASE. Methods that enhance HH yields by orders of magnitude have been demonstrated [23, 24], although the impact of these methods on the attosecond character of the source has not been investigated) It was assumed that the wavelength of the Ti:Sapphire drive laser was $\lambda_{d}=780 \mathrm{~nm}$ and the FEL was then tuned to be resonant with the $63 \mathrm{rd}$ harmonic of the drive laser with $\lambda_{r}=12.4 \mathrm{~nm}$. The repetition rate of the seed pulses is the same as that of the drive laser which could be expected to rise from the current 10 's $\mathrm{Hz}$ to $\sim 1 \mathrm{kHz}$ in the next few years [22].

Each undulator module had 4 periods of $3.1 \mathrm{~cm}$ and each chicane had a delay of 28 radiation wavelengths corresponding to a slippage enhancement $S_{e}=8$. These parameters for the undulator-chicane modules then match the mode spacing of the $\mathrm{HH}$ seed. The amplified seed approaching saturation, after 47 modules, is plotted in Fig. 8 and the pulse temporal and spectral properties agree well with the equivalent one-dimensional $S_{e}=8.125$ case of Fig. 6 . The FWHM length of each pulse in the 

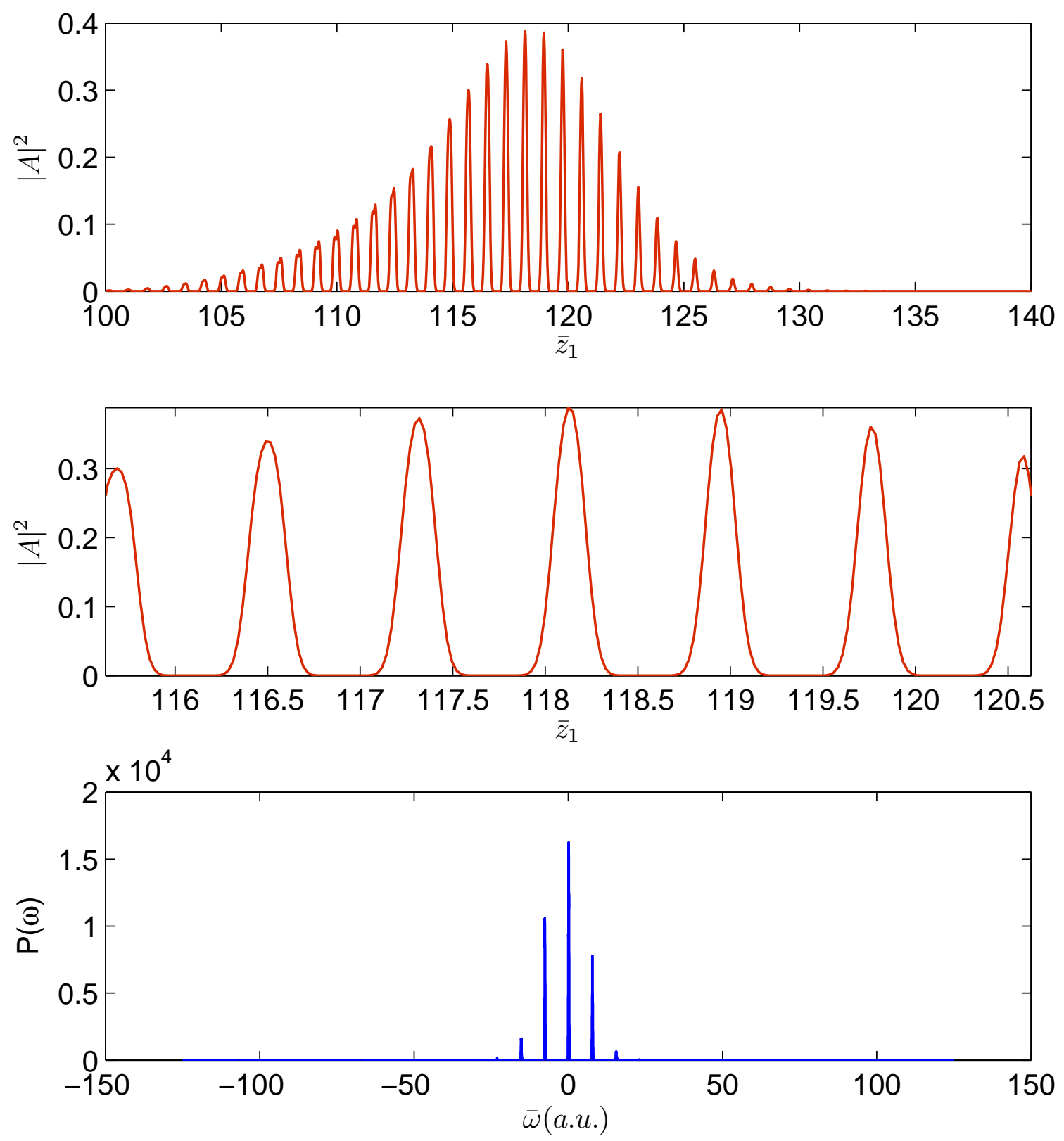

Figure 6. Scaled longitudinal intensity profile (top and middle) and scaled spectral power distribution of the amplified $\mathrm{HH}$ radiation for $\bar{l}=0.101\left(S_{e}=8.125\right)$ and an initial energy spread $\sigma_{\gamma} / \gamma=0.02 \%$, after 42 undulator-chicane modules.

amplified train is 300 as. Further 3D simulations (not shown) with $S_{e}=4$ agreed well with the $S_{e}=4.0625$ case of Fig. 3. As may be expected from an amplifier seeded using a source of good temporal coherence with power well above noise level, the temporal coherence length of the amplified output is found, though not shown here, to be retained with little reduction. 

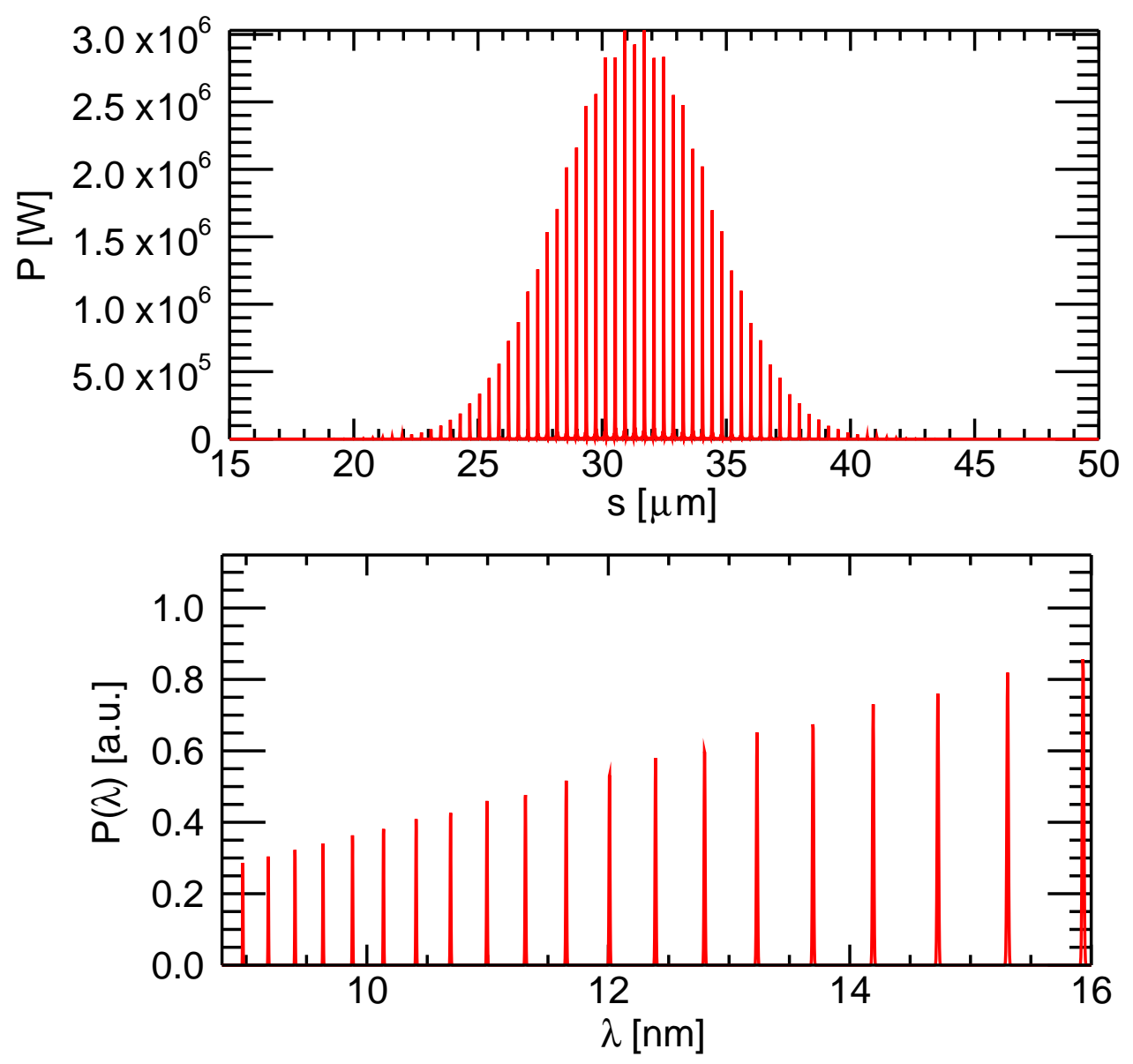

Figure 7. Longitudinal intensity profile (top) and spectral power distribution (bottom) of the HH seed in 3D simulations.

\section{Conclusions}

It was shown, using $1 \mathrm{D}$ and $3 \mathrm{D}$ simulations, that the attosecond structure of the $\mathrm{HH}$ seed can be amplified to saturation using a Mode Locked Optical Klystron FEL amplifier configuration of [16] to increase the peak power by a factor $\sim 300$. No pre-conditioning of the electron beam (e.g. energy or current modulation) was required. The only requirement is for an undulator-chicane modular structure, the radiation spectrum of which matches an $\mathrm{HH}$ seed source.

The maximum bandwidth of the amplified radiation is critical to achieving the minimum pulse durations (maximum mode number) from the system, and is set by the single undulator module spectrum. Reducing the number of undulator periods per module therefore appears to be the most effective route to achieving the shortest possible pulse durations. The introduction of a modulation to the electron bunch at the modespacing may well restrict the slow pulse broadening effect observed and so allow shorter pulse durations than those reported here. This will be investigated in future work. The behaviour of the MLOK system when modelled in a code allowing a greater spectral bandwidth, and so shorter pulse lengths, such as that of [25] will also be investigated. 

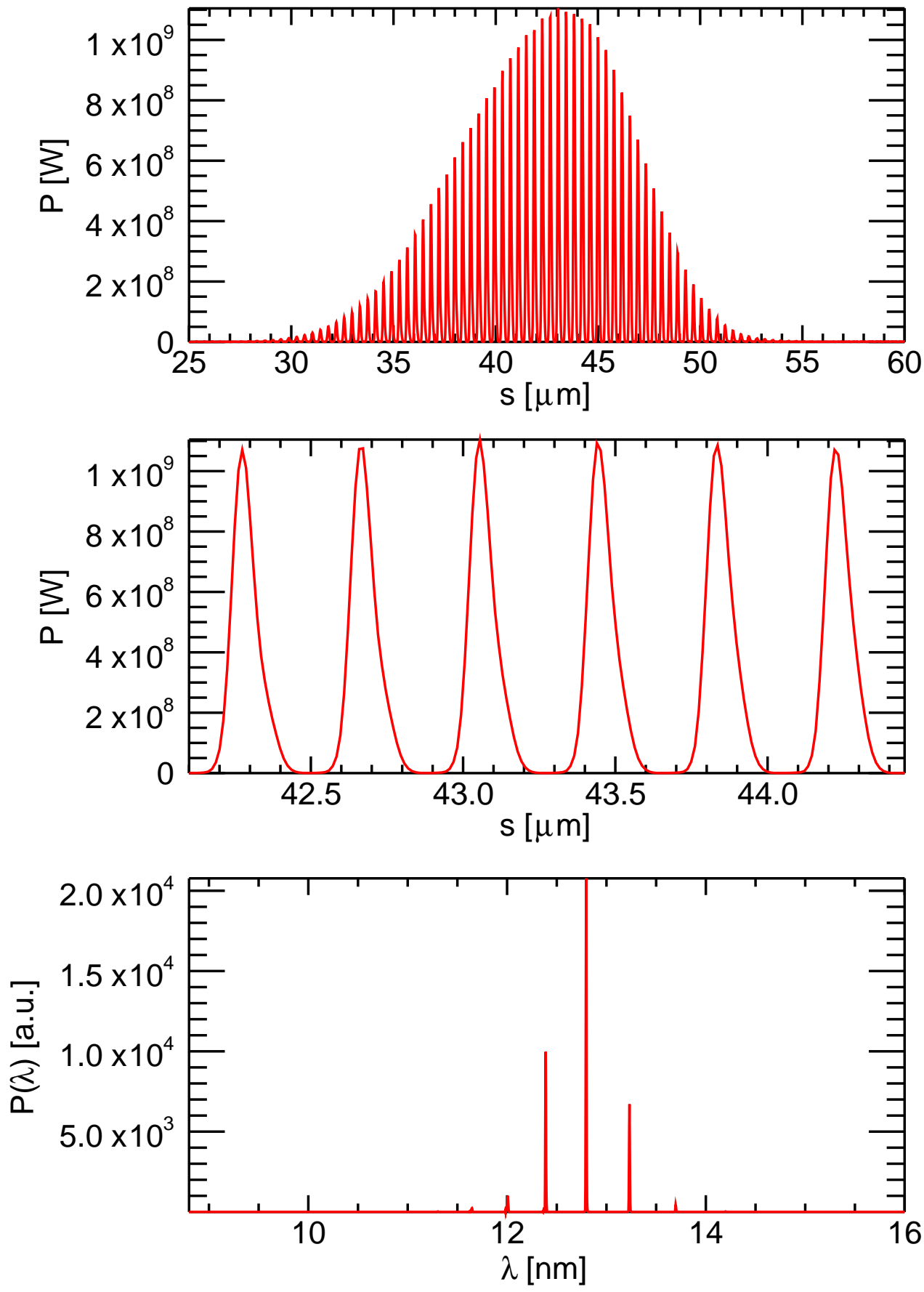

Figure 8. Longitudinal intensity profile (top and middle) and spectral power distribution (bottom) of the amplified $\mathrm{HH}$ radiation in 3D simulations, with $S_{e}=8$. The agreement with the equivalent 1D simulations shown in Fig. 6 is very good.

\section{Acknowledgments}

This work received support from Brookhaven Science Associates, LLC under Contract No. DE-AC02-98CH10886 with the U.S. Department of Energy. 


\section{References}

[1] Corkum P B and Krausz F 2007 Nature Physics 3381

[2] Krausz F and Ivanov M 2009 Rev. Mod. Phys. 81163

[3] Johnsson P, López-Martens R, Kazamias S, Mauritsson J, Valentin C, Remetter T, Varjú K, Gaarde M B, Mairesse Y, Wabnitz H, Salières P, Balcou Ph, Schafer K J and L'Huillier A 2005 Phys. Rev. Lett 95013001

[4] Remetter T, Johnsson P, Mauritsson J, Varjú K, , Ni Y, Lépine F, Gustafsson E, Kling M, Khan J, López-Martens R, Schafer K J, Vrakking M J J and L'Huillier A 2006 Nature Physics 2 323-326

[5] Varjú K, Johnsson P, Mauritsson J, Remetter T, Ruchon T, Ni Y, Lépine F, Kling M, Khan J, Schafer K J, Vrakking M J J and L'Huillier A 2006 J. Phys. B: At. Mol. Opt. Phys. 3939833991

[6] Mauritsson J, Johnsson P, Mansten E, Swoboda M, Ruchon T, L'Huillier A and Schafer K J 2008 Phys. Rev. Lett 100073003

[7] Klaiber M, Hatsagortsyan K Z, Müller C and Keitel C H 2008 Optics Lett. 33 411-413

[8] Singh K P, He F, Ranitovic P, Cao W, De S, Ray D, Chen S, Thumm U, Becker A, Murnane M M , Kapteyn H C, Litvinyuk I V and Cocke C L 2010 Phys. Rev. Lett. 104023001

[9] Bonifacio R, Pellegrini C and Narducci L 1984 Optics Commun. 50373

[10] McNeil B W J, Clarke J A, Dunning D J, Hirst G J, Owen H L, Thompson N R, Sheehy B and Williams P H 2007 New Journal of Physics 982

[11] Xiang D, Huang Z and Stupakov G 2009 Phys. Rev. ST Accel. Beams 12060701 and refs. [6-15] therein

[12] Ding Y et al 2009 Phys. Rev. ST Accel. Beams 12060703

[13] Penn G and Zholents A 2009 Proceedings of the 31st International Free Electron Laser Conference, Liverpool, U.K. MOPC73 p 176

[14] Ding Y et al 2009 Phys. Rev. Lett. 102254801

[15] McNeil B W J and Thompson N R 2010 Proceedings of the 32nd International Free Electron Laser Conference, Malmo, Sweden THOB4

[16] Thompson N R and McNeil B W J 2008 Phys. Rev. Lett. 100203901

[17] Siegman A E 1986 Lasers (University Science Books, Sausalito, USA) Ch. 27

[18] L'Huillier A and Balcou Ph 1993 Phys. Rev. Lett. 70774

[19] Antoine Philippe, L'Huillier Anne and Lewenstein Maciej 1996 Phys. Rev. Lett. 771234

[20] McNeil B W J, Robb G R M, Dunning D and Thompson N R 2006 Proceedings of the 28th Int. FEL Conference, Berlin, Germany p 59

[21] Reiche S 1999 Nucl. Inst. Meth. Phys. Res. A 429243

[22] Marangos J et al. 2010 NLS Project: Conceptual Design Report Section 10.2 available from: http://www.newlightsource.org

[23] Jong Kim I et al. 2005 Phys. Rev. Lett. 94243901

[24] Takahashi E J et al 2007 Phys. Rev. Lett. 99053904

[25] McNeil B W J and Robb G R M 2002 Phys. Rev. E 65046503 